\title{
THE STRONG-BOUNDED TOPOLOGY ON GROUPS OF AUTOMORPHISMS OF A VON NEUMANN ALGEBRA
}

\author{
ROBERT R. KALLMAN ${ }^{1}$
}

0 . Introduction. Let $R$ be a von Neumann algebra on the Hilbert space $H, G$ a topological group, and $a \rightarrow \varphi(a)$ a representation of $G$ as a group of $*$-automorphisms of $\boldsymbol{R}$. Recall that $a \rightarrow \varphi(a)$ is continuous in the strong-bounded topology if and only if

$$
\sup _{T \in R,\|T\| \leqq 1}\|[\varphi(a)(T)-T] x\| \rightarrow 0 \quad(a \rightarrow e)
$$

for all $x \in H$. The purpose of this note is to show that for certain von Neumann algebras $R$ and certain groups of $*$-automorphisms $\varphi(a)$, the continuity of $\varphi(a)$ in the strong-bounded topology is a very restrictive condition. For example, if $\boldsymbol{R}$ is abelian and $s \rightarrow \varphi(s)$ is a one-parameter group of $*$-automorphisms of $\boldsymbol{R}$, continuous in the strong-bounded topology, then $\varphi(s)$ is the identity automorphism for all $s$. If $R$ is either a $I_{\infty}$ factor or a $I I I_{\infty}$ factor (and $H$ is separable), then a strong-bounded continuous one-parameter group of inner automorphisms must be uniformly continuous. Hence, strongbounded continuous groups of automorphisms are probably not useful in quantum field theory, since the corresponding Hamiltonian operators have bounded spectrum.

The author would like to thank L. Bongaarts for a number of stimulating conversations on the role of automorphisms of von Neumann algebras in quantum field theory.

The notation and terminology of this note is that of Dixmier [1].

1. The results. We make two preliminary remarks before going on to the main theorems.

First, note that if $x \in H$ is cyclic for $R^{\prime}$ and

$$
\sup _{T \in R,\|T\| \leqq 1}\|[\varphi(a)(T)-T] x\| \rightarrow 0 \quad(a \rightarrow e),
$$

then $a \rightarrow \varphi(a)$ is continuous in the strong-bounded topology.

The second remark is contained in the following lemma.

Lemma 1.1. Let $\boldsymbol{R}$ be a von Neumann algebra on $H, G$ a topological group, and $a \rightarrow \varphi(a)$ a representation of $G$ as a group of $*$-automorphisms

Received by the editors January 17, 1969.

1 Massachusetts Institute of Technology, Cambridge, Massachusetts 02139; supported in part by N.S.F. grant GP-9141. 
of $R$, continuous in the strong-bounded topology. Let $\mathbf{S}$ be a von Neumann algebra and $\phi: R \rightarrow S a$-isomorphism. Then $a \rightarrow \phi \circ \varphi(a) \circ \phi^{-1}$ is a representation of $G$ as a group of *-automorphisms of $S$, continuous in the strong-bounded topology.

Proof. By Dixmier [1, Théoréme 3, p. 58], it suffices to consider three special cases: (1) $\phi$ is an ampliation $\phi: T \rightarrow T \otimes I$, where $I$ is the identity on some Hilbert space $K$; (2) $\phi$ is an induction, $\phi: T \rightarrow T_{E}$, where $E$ is a projection in $R^{\prime}$ with central support equal to $I$; (3) $\phi$ is a spatial isomorphism. Cases (2) and (3) are easy and are left to the reader. Case (1) may be reformulated as follows. There exists some index set $A$ such that $H \otimes K=\sum_{\alpha \in A} \oplus H_{\alpha}$, where for all $\alpha H_{\alpha}$ is a copy of $\boldsymbol{H}$. $\phi$ then has the form $\phi: T \in R \rightarrow \sum_{\alpha \in A} \oplus T_{\alpha} \in R \otimes I$, where $T_{\alpha}=T$ for all $\alpha$. Given $x \in H \otimes K$, choose indices $\alpha_{1}, \cdots, \alpha_{n}$ such that $x_{\alpha_{1}} \oplus \cdots \oplus x_{\alpha_{n}}$ is as close in norm to $x$ as desired. Here $x_{\beta}$ is the $\beta$ th component of $x$. Then choose $\delta$ so that

$$
\sup _{|s| \leqq \delta, T \in R,\|T\| \leqq 1,1 \leqq i \leqq n} \|[\varphi(s)(T)-T] x_{\alpha_{i} \|}
$$

is as small as desired. Simple inequalities complete the proof. Q.E.D.

THEOREM 1.2. Suppose $R$ is an abelian von Neumann algebra and $s \rightarrow \varphi(s)$ is a one-parameter group of $*$-automorphisms of $\boldsymbol{R}$, continuous in the strong bounded topology. Then $\varphi(s)$ is the identity automorphism for all $s$.

Before proving Theorem 1.2, we make a few preliminary remarks and prove a preliminary lemma.

Let $\varphi$ be a $*$-automorphism of $R$. Recall that $\varphi$ is freely acting on $R$ if, given a nonzero projection $P$ in $R$, there exists a nonzero projection $Q$ in $R$ such that $Q \leqq P$ and $Q \perp \varphi(Q)$. In general, there exist projections $P$ and $Q$, fixed under $\varphi$, such that $\varphi$ is the identity on $R_{Q}$ and is freely acting on $\boldsymbol{R}_{P}$. For each $\varphi(s)$, let $P_{s}$ and $Q_{s}$ be the corresponding $P$ and $Q$. Note that $P_{s}=P_{-s}$ and $Q_{s}=Q_{-s}$. Theorem 1.2 will be proved if we can show $P_{s}=0$ for all $s$.

Every abelian von Neumann algebra is *-isomorphic to a maximal abelian von Neumann algebra. Hence, by Lemma 1.1 it suffices to consider the case in which $R$ is maximal abelian. In this case the spectral theorem states that $R$ is unitarily equivalent to the multiplication algebra of some measure space $(M, \mu)$. From now on we assume that $R$ is such a multiplication algebra.

Next, a lemma needed in the proof of Theorem 1.2. 
Lemma 1.3. Let $(\Omega, \nu)$ be a measure space, $f \in L^{2}(\Omega, \nu)$, and $\varphi$ a freely acting *-automorphism of $L^{\infty}(\Omega, \nu)$. Then there exists a projection $P \in L^{\infty}(\Omega, \nu)$ such that $P \perp \varphi(P)$ and $\|P f\|_{2} \geqq \frac{1}{3}\|f\|_{2}$.

Proof. By Zorn's Lemma, choose a maximal projection $P$ such that $P \perp \varphi(P) . P$ is nonzero since $\varphi$ is freely acting on $L^{\infty}(\Omega, \nu)$. Let $Q=P+\varphi(P)$. Claim $\varphi(I-Q) \leqq P$. To show this it suffices to show that $\varphi(I-Q) \leqq Q$. If $R=\varphi(I-Q) \cdot(I-Q) \neq 0$, then $\varphi^{-1}(R) \leqq I-Q \perp Q$ and $R \leqq I-Q \perp Q$. Since $\varphi$ is freely acting, there exists a nonzero projection $S \leqq \varphi^{-1}(R)$ such that $S \perp \varphi(S)$. But $S \leqq \varphi^{-1}(R) \perp Q$ and $\varphi(S) \leqq R \perp Q$. This contradicts the maximality of $P$. Hence, $\varphi(I-Q) \leqq P$. Now $I=P+\varphi(P)+(I-Q)$. At least one of $\|P f\|_{2},\|\varphi(P) f\|_{2}$, and $\|(I-Q) f\|_{2}$ is greater than or equal to $\frac{1}{3}\|f\|_{2}$. But $I-Q \perp \varphi(I-Q), P \perp \varphi(P)$, and $\varphi(P) \perp \varphi^{2}(P)$. The result follows.

Q.E.D.

Easy examples show that the constant $\frac{1}{3}$ in the above lemma is best possible.

Proof of Theorem 1.2. Let $s$ be a positive real number, $Q$ is a projection in $L^{\infty}(M, \mu)$ corresponding to a measurable subset of $M$ of finite measure, and $\chi_{Q}$ the characteristic function of $Q$. Now $\left(P_{s}, \mu\right)$ is a measure space and $\varphi(s)$ is a freely acting $*$-automorphism of $L^{\infty}\left(P_{s}, \mu\right) L^{\infty}(M, \mu)$. By Lemma 1.3 there exists a projection $R \in L^{\infty}\left(P_{s}, \mu\right)$ such that

$$
\left[\int_{P_{s}} R \chi_{Q} d \mu\right]^{\frac{1}{2}} \geqq \frac{1}{3}\left[\int_{P_{s}} \chi_{Q} d \mu\right]^{\frac{1}{2}}
$$

and $R \perp \varphi(s)(R)$.

$$
\begin{aligned}
\|[\varphi(s)(R)- & R] \chi_{Q} \|_{2} \\
& =\left\|P_{s}[\varphi(s)(R)-R]_{\chi_{Q}}\right\|_{2}=\left[\int_{P_{s}}|\varphi(s)(R)-R|{ }^{2} \chi_{Q} d \mu\right]^{\frac{1}{2}} \\
& =\left[\int_{P_{s}}\left[\varphi(s)(R) \chi_{Q}+R \chi_{Q}\right] d \mu\right]^{\frac{1}{2}} \geqq\left[\int_{P_{s}} R \chi_{Q} d \mu\right]^{k} \\
& \geqq \frac{1}{3}\left[\int_{P_{s}} \chi_{Q} d \mu\right]^{\frac{1}{3}}=\frac{1}{3} \mu\left(P_{s} Q\right)^{\frac{1}{2}} .
\end{aligned}
$$

Hence,

$$
9 \sup _{|r| \leqq s, T \in R, \| T \rrbracket \leqq 1}\left\|[\varphi(r)(T)-T] \chi_{Q}\right\|_{2}^{2} \geqq \mu\left(P_{8} Q\right) .
$$

If $\varphi$ is a *-automorphism of $R$ and $\varphi$ leaves a projection $R$ absolutely fixed, then $\varphi^{n}$ leaves $R$ absolutely fixed $(n \geqq 1)$. Hence, $Q_{s / n} \leqq Q_{s}$ and therefore $P_{s / n} \geqq P_{s}$. From this it follows that 


$$
\begin{aligned}
\mu\left(P_{s} Q\right) & \leqq \mu\left(P_{s / n} Q\right) \\
& \leqq 9 \sup _{|r| \leqq s / n, T \in R,\|T\| \leqq 1}\left\|[\varphi(r)(T)-T] \chi_{Q}\right\|_{2}^{2} \rightarrow 0 \quad(n \uparrow+\infty) .
\end{aligned}
$$

Hence, $P_{s}$ is or thogonal to $Q$. Since $Q$ 's corresponding to measurable sets of finite measure generate $R, P_{s}=0$ for all $s$.

As noted above, this proves Theorem 1.2.

Q.E.D.

Theorem 1.4. Suppose $R$ is either: (1) a $I_{\infty}$ factor; or (2) $H$ is separable and $R$ is a $I I I_{\infty}$ factor. Let $s \rightarrow U(s)$ be a strongly continuous oneparameter unitary group in $R$. Let $\varphi(s)(T)=U(s) T U(-s)(T \in R)$. Suppose $\varphi(s)$ is continuous in the strong-bounded topology. Then $s \rightarrow U(s)$ is uniformly continuous.

Proof. Assume $R$ is a $I_{\infty}$ factor. Let $U(s)=e^{i s A}$. $U(s)$ is uniformly continuous if and only if $A$ is bounded. If $U(s)$ is not uniformly continuous, assume that the spectrum of $A$ is unbounded on the positive real axis (otherwise consider the one-parameter unitary group $V(s)=U(-s))$. Let $U(s)=\int_{-\infty}^{+\infty} e^{i \lambda s} d E(\lambda)$. Then there exist integers $n_{0}<n_{1}<\cdots \uparrow+\infty$ such that $E\left(\left[n_{k}, n_{k}+1\right)\right) \neq 0(k \geqq 0)$. Choose a minimal projection $P_{0} \leqq E\left(\left[n_{0}, n_{0}+1\right)\right)$ and $x \in P_{0}(H),\|x\|=1$. Choose partial isometrics $U_{k}(k \geqq 1)$ such that $U_{k}^{*} U_{k}=P_{0}$ and $P_{k}=U_{k} U_{k}^{*} \leqq E\left(\left[n_{k}, n_{k}+1\right)\right)$. Now since multiplication on the right by $U(s)$ carries the unit ball of $R$ onto itself

$$
\begin{aligned}
\sup _{T \in R,\|T\| \leqq 1} & \|[U(s) T U(-s)-T] x\|=\sup _{T \in R,\|T\| \leqq 1}\|[U(s) T-T U(s)] x\| . \\
\left.\| \mid U(s) U_{k}-U_{k} U(s)\right] x \| & \\
\geqq & \| \exp \left(i n_{k} s\right) E\left(\left[n_{k}, n_{k}+1\right)\right) U_{k} x-\exp \left(i n_{0} s\right) U_{k} E\left(\left[n_{0}, n_{0}+1\right) x \|\right. \\
& \quad-\left\|U(s) U_{k} x-\exp \left(i n_{k} s\right) E\left(\left[n_{k}, n_{k}+1\right)\right) U_{k} x\right\| \\
& \quad-\left\|U_{k} \exp \left(i n_{0} s\right) E\left(\left[n_{0}, n_{0}+1\right)\right) x-U_{k} U(s) x\right\| \\
\geqq & \left|\exp \left(i n_{k} s\right)-\exp \left(i n_{0} s\right)\right|-\sup _{\lambda \in\left[n_{k}, n_{k}+1\right]}\left|\exp (i \lambda s)-\exp \left(i n_{k} s\right)\right| \\
& \quad-\sup _{\lambda \in\left[n_{0}, n_{0}+1\right]}\left|\exp (i \lambda s)-\exp \left(i n_{0} s\right)\right| \\
= & \left|\exp \left(i\left(n_{k}-n_{0}\right) s\right)-1\right|-2 \sup _{\lambda \in[0,1]}|\exp (i \lambda s)-1| .
\end{aligned}
$$

Now $n_{k}-n_{0} \uparrow+\infty$ as $k \uparrow+\infty$. Hence,

$$
\limsup _{s \rightarrow 0, T \in R,\|T\| \leqq 1}\|[U(s) T U(-s)-T] x\|=2 .
$$

Contradiction. Hence, $U(s)$ must be uniformly continuous. 
Now assume that $R$ is a $I I I_{\infty}$ factor on a separable Hilbert space. The proof of the theorem for this case follows the proof of the $I_{\infty}$ case almost verbatim. The only twist is the use of the fact that two nonzero projections in a $I I I_{\infty}$ factor on a separable Hilbert space are equivalent in the Murray-von Neumann sense. The details are left to the reader.

Q.E.D.

We remark that if $R$ is a $I_{\infty}$ factor on a separable Hilbert space, then the following apparent strengthening of Theorem 1.4 is true. If $\varphi(s)$ is an arbitrary one-parameter group of $*$-automorphisms of $R$, continuous in the strong-bounded topology, then $s \rightarrow \varphi(s)$ is continuous in the norm topology. A sketch of the proof of this follows. $\varphi(s)$ is inner for each $s$ since $R$ is a $I_{\infty}$ factor. $\varphi(s)$ continuous in the strongbounded topology implies that $\varphi(s)$ is continuous in the weakbounded topology. Since $H$ is separable, a result of Kadison ([2, Theorem 4.13, p. 195]) now shows that there exists a strongly continuous one-parameter unitary group $s \rightarrow U(s)$ in $\boldsymbol{R}$ such that $\varphi(s)(T)$ $=U(s) T U(-s)$. Theorem 1.4 shows $s \rightarrow U(s)$ is uniformly continuous. Easy estimates now imply that $s \rightarrow \varphi(s)$ is continuous in the norm topology.

It is unknown to the author whether or not an analogue of Theorem 1.4 holds for $R$ a $I I_{\infty}$ factor. The $I I_{1}$ case is handled by the next theorem.

Theorem 1.5. Let $R$ be a $I I_{1}$ von Neumann algebra and $U(s)$ a strongly continuous one-parameter unitary group in $\boldsymbol{R}$. Let $\varphi(s)(T)$ $=U(s) T U(-s)$. Then $s \rightarrow \varphi(s)$ is continuous in the strong-bounded topology.

Proof. There exists some index set $B$ such that $\boldsymbol{R}=\sum_{\beta \in B} \oplus \boldsymbol{R}_{\beta}$, where each $\boldsymbol{R}_{\boldsymbol{\beta}}$ has a faithful finite trace. Note that each $\varphi(s)$ leaves Cent $R$ fixed and hence $\varphi(s)=\sum_{\beta \in B} \oplus \varphi_{\beta}(s)$, where $\varphi_{\beta}(s)$ is a oneparameter group of $*$-automorphisms of $\boldsymbol{R}_{\beta}$. An argument like that used in the proof of Lemma 1.1 shows that $\varphi(s)$ is continuous in the strong-bounded topology if each $\varphi_{\beta}(s)$ is. Hence, we may assume $R$ has a faithful finite trace. By Lemma 1.1, we may assume $R$ has a trace vector $x$. By the remarks preceeding Lemma 1.1, it suffices to show that

$$
\begin{aligned}
& \sup _{T \in R,\|T\| \leqq 1}\|[\varphi(s)(T)-T] x\| \rightarrow 0 \quad(s \rightarrow 0) ; \\
& \text { i.e., } \quad \sup _{T \in R,\|T\| \leqq 1}\|\varphi(s)(T)-T\|_{2} \\
& =\|U(s) T-T U(s)\|_{2} \rightarrow 0 \quad(s \rightarrow 0) \\
& \text { (here }\|\cdot\|_{2} \text { denotes the trace norm). }
\end{aligned}
$$


Let $P$ be a "large" spectral projection of

$$
U(s)=\int_{-\infty}^{+\infty} e^{i \lambda s} d E(\lambda) \quad(\text { say } P=E([-n, n]) \text { for large } n)
$$

such that $\|I-P\|_{2}<\epsilon$. Let $T \in R,\|T\| \leqq 1$. Then

$$
\begin{aligned}
\|\varphi(s)(T)-T\|_{2} & \leqq\|P U(s) P T P-P T P U(s) P\|_{2}+6 \epsilon \\
& \leqq 2\|P U(s) P-P\|_{\infty}+6 \epsilon .
\end{aligned}
$$

$\|P U(s) P-P\|_{\infty} \rightarrow 0(s \rightarrow 0)$ since $P U(s) P$ is a unitary operator with bounded spectrum on $P(H)$. Hence, $\|\varphi(s)(T)-T\|_{2} \rightarrow 0(s \rightarrow 0)$.

We note that Theorem 1.5 is not true for an arbitrary one-parameter group $\varphi(s)$ of $*$-automorphisms of a $I I_{1}$ factor. For example, for $n \geqq 1$, let $\boldsymbol{R}_{n}$ be the algebra of all $2 \times 2$ matrices, $\tau_{n}$ the normalized trace on $\boldsymbol{R}_{n}$, and $\varphi_{n}(s)$ the $*$-automorphism of $\boldsymbol{R}_{n}$ given by the unitary $e_{0}^{i n s} 0_{e-i n s}$. Further, let $S$ be the $C^{*}$-tensor product $\otimes_{n \geqq 1} R_{n}, \tau=\bigotimes_{n \geqq 1} \tau_{n}$,

$$
\left(\begin{array}{cc}
e^{i n s} & 0 \\
0 & e^{-i n s}
\end{array}\right)
$$

and $\varphi(s)=\otimes_{n \geq 1} \varphi_{n}(s)$. If $\Pi_{\tau}$ is the cyclic representation of $S$ with cyclic vector $\xi_{\tau}$ on the Hilbert space $H_{\tau}$ corresponding to the state $\tau$, $\Pi_{\tau}$ is faithful, and $\Pi_{\tau}(S)^{\prime \prime}$, the strong closure of $\Pi_{\tau}(S)$, is a $I I_{1}$ factor. Since $\varphi(s)$ leaves $\tau$ invariant, there exists a strongly continuous oneparameter unitary group $U(s)$ on $\boldsymbol{H}_{\tau}$ such that

$$
L(s) \Pi(T) U(-s)=\Pi(\varphi(s)(T)) \quad(T \in \mathbf{S}) .
$$

Easy computations show that

$$
\lim _{s \rightarrow 0, T \in \Pi_{\tau}(s),\|T\| \leqq 1}\left\|[U(s) T U(-s)-T] \xi_{\tau}\right\|=2 .
$$

\section{BIBLIOGRAPHY}

1. J. Dixmier, Les Algebres D'operateurs dahs L'espace Hilbertien, Cahiers Sci., Fasc. XXV Gauthier-Villars, Paris, 1957.

2. R. V. Kadison, "Transformations of states in operator theory and dynamics," Topology, 3, Supplement 2, (1965) 177-198.

Massachusetts Institute of Technology 